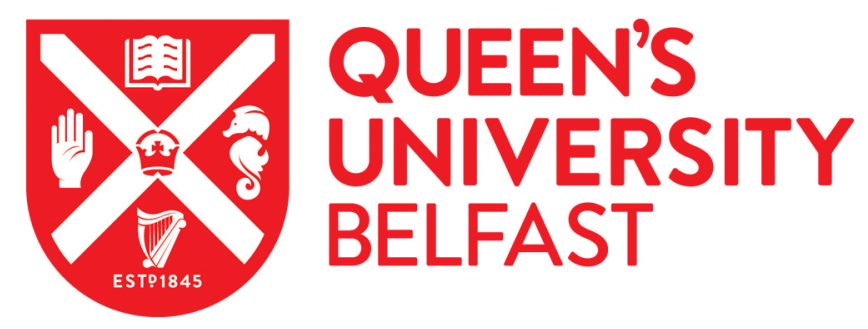

\title{
CONSEQUENCES of A DISTANT MASSIVE PLANET on the LARGE SEMIMAJOR AXIS TRANS-NEPTUNIAN OBJECTS
}

Shankman, C., Kavelaars, J. J., Lawler, S. M., Gladman, B. J., \& Bannister, M. T. (2017). CONSEQUENCES of A DISTANT MASSIVE PLANET on the LARGE SEMIMAJOR AXIS TRANS-NEPTUNIAN OBJECTS.

Astronomical Journal, 153(63), 1-9. https://doi.org/10.3847/1538-3881/153/2/63

Published in:

Astronomical Journal

Document Version:

Publisher's PDF, also known as Version of record

Queen's University Belfast - Research Portal:

Link to publication record in Queen's University Belfast Research Portal

Publisher rights

(C) 2017. The American Astronomical Society. All rights reserved.This work is made available online in accordance with the publisher's policies. Please refer to any applicable terms of use of the publisher.

\section{General rights}

Copyright for the publications made accessible via the Queen's University Belfast Research Portal is retained by the author(s) and / or other copyright owners and it is a condition of accessing these publications that users recognise and abide by the legal requirements associated with these rights.

Take down policy

The Research Portal is Queen's institutional repository that provides access to Queen's research output. Every effort has been made to ensure that content in the Research Portal does not infringe any person's rights, or applicable UK laws. If you discover content in the Research Portal that you believe breaches copyright or violates any law, please contact openaccess@qub.ac.uk. 


\title{
CONSEQUENCES OF A DISTANT MASSIVE PLANET ON THE LARGE SEMIMAJOR AXIS TRANS- NEPTUNIAN OBJECTS
}

\author{
C. Shankman ${ }^{1}$, J. J. Kavelaars ${ }^{2}$, S. M. Lawler ${ }^{2}$, B. J. Gladman ${ }^{3}$, and M. T. Bannister ${ }^{4}$ \\ ${ }^{1}$ Department of Physics and Astronomy, University of Victoria, Elliott Building, \\ 3800 Finnerty Road, Victoria, BC V8P 5C2, Canada; cshankm@uvic.ca \\ ${ }^{2}$ National Research Council of Canada, 5071 West Saanich Road, Victoria, BC, V9E 2E7, Canada \\ ${ }^{3}$ Department of Physics and Astronomy, The University of British Columbia, 6224 Agricultural Road, Vancouver, BC, V6T 1Z1, Canada \\ ${ }^{4}$ Astrophysics Research Centre, School of Mathematics and Physics, Physics Building, Queen's University Belfast, Belfast, County Antrim, \\ BT7 1NN, United Kingdom \\ Received 2016 October 11; revised 2016 December 6; accepted 2016 December 8; published 2017 January 11
}

\begin{abstract}
We explore the distant giant planet hypothesis by integrating the large-semimajor-axis, large-pericenter transNeptunian objects (TNOs) in the presence of the giant planets and an external perturber whose orbit is consistent with the proposed distant, eccentric, and inclined giant planet, so-called planet 9 . We find that TNOs with semimajor axes greater than 250 au experience some longitude of perihelion shepherding, but that a generic outcome of such evolutions is that the TNOs evolve to larger pericenter orbits and commonly get raised to retrograde inclinations. This pericenter and inclination evolution requires a massive disk of TNOs (tens of $M_{\oplus}$ ) in order to explain the detection of the known sample today. Some of the highly inclined orbits produced by the examined perturbers will be inside of the orbital parameter space probed by prior surveys, implying a missing signature of the ninth-planet scenario. The distant giant planet scenarios explored in this work do not reproduce the observed signal of simultaneous clustering in argument of pericenter, longitude of the ascending node, and longitude of perihelion in the region of the known TNOs.
\end{abstract}

Key words: Kuiper belt: general - planet-disk interactions

Supporting material: animation

\section{INTRODUCTION}

In solar system studies, the observation of unexpected orbital behavior has been used to further our understanding of both physics and the composition of the solar system. Two exemplary successes of this process can be found in the prediction of the existence of Neptune from observing the evolving orbit of Uranus (Le Verrier 1846), and the confirmation of general relativity through its ability to explain the precession of Mercury's orbit (Le Verrier 1859; Einstein 1916). This pattern repeats itself in the outer solar system today as we discover new mysteries in the trans-Neptunian object (TNO) population.

The distant perihelion of $2001 \mathrm{CR}_{105}$ (Gladman et al. 2002) demonstrated that some TNOs inhabit the space beyond the domain that is dominated by the gravitational influence of Neptune. With a large semimajor axis $a$ of 226 astronomical units (au) and a pericenter $q$ of $44 \mathrm{au}$, well beyond the $q \lesssim 37 \mathrm{au}$ active influence of Neptune (Lykawka \& Mukai 2007; Gladman et al. 2008, pp. 43-57), $2001 \mathrm{CR}_{105}$ required a new dynamical process to emplace TNOs on orbits so separated from the influence of the giant planets. Gladman et al. (2002) explore various possible formation scenarios for $2001 \mathrm{CR}_{105}$, including a fossilized disk, a passing star, the presence of a small (lunar- to Mars-sized) planet beyond the orbit of Neptune, and others argue that $2001 \mathrm{CR}_{105}$ can be emplaced on its orbit by a complex path of planetary migration and resonance capture, but only for objects with $a<260$ au (Gomes 2003; Gomes et al. 2005). The discovery of 2001 $\mathrm{CR}_{105}$ was followed by surveys finding other TNOs with orbits outside the gravitational domain of Neptune (now numbering in the tens) that have revealed unexpected and difficult-toexplain structure in the outer solar system.
With the discovery of (90377) Sedna (Brown et al. 2004), an even more extreme orbit space was revealed. Sedna, with its $q$ at $76 \mathrm{au}$ and $a$ of $500 \mathrm{au}$, is not dynamically coupled to the giant planets or to galactic tides. It is difficult to form planetesimals at such a large distance given current models of planetesimal formation, and therefore the current orbits of these detached TNOs require some dynamical interaction (ongoing or long past) to have emplaced them on such orbits. Theories for emplacement of TNOs on large- $q$ orbits have included an additional planet in the solar system (e.g., Gladman et al. 2002; Brown et al. 2004; Gomes et al. 2006; Soares \& Gomes 2013), stellar flybys (e.g., Ida et al. 2000; Kenyon \& Bromley 2004; Morbidelli \& Levison 2004; Kaib et al. 2011; Soares \& Gomes 2013; Brasser \& Schwamb 2015), and ejected rogue planets (e.g., Thommes et al. 2002; Gladman \& Chan 2006). These discoveries have continued, with one new extreme orbit TNO discovered every few years (e.g., $2004 \mathrm{VN}_{112}$, Becker et al. 2008; $2010 \mathrm{~GB}_{174}$, Chen et al. 2013).

In 2014, Trujillo \& Sheppard (2014) reported the discovery of another large-pericenter TNO, $2012 \mathrm{VP}_{113}$, with $q \simeq 80$. Trujillo \& Sheppard (2014) also noted a clustering (i.e., apparent grouping in the observed sample) of pericenter arguments $\omega$ near $0^{\circ}$ for all of the known TNOs having $a>150$ au and $q>30$ au (see Tables 1 and 2 for listing and uncertainties). There are known detection biases for discovering objects at their pericenters and in the ecliptic plane, which could explain an enhancement of detections of objects with $\omega=180^{\circ}$ versus $\omega=0^{\circ}$; however, there have been no TNOs detected with $\omega$ near $180^{\circ}$. As of this writing, there have been no published demonstrations that the lack of $\omega=180^{\circ}$ TNOs results from an observation bias, nor has there been a demonstration that these detections should be free from such 

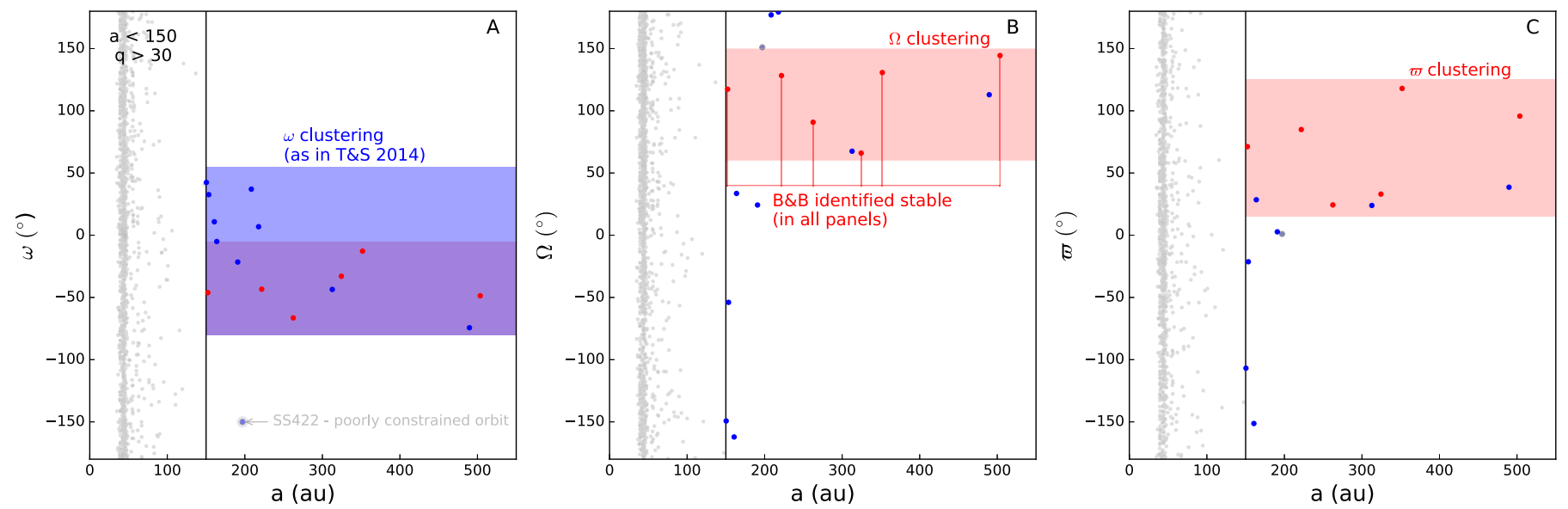

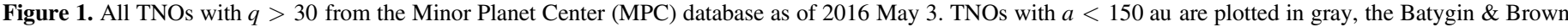

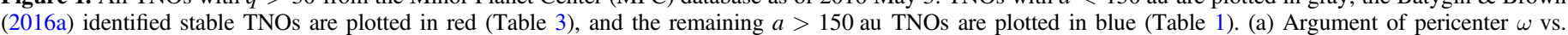

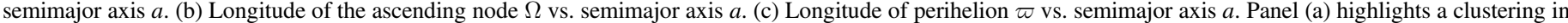

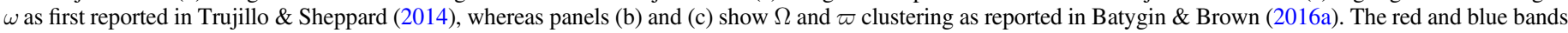

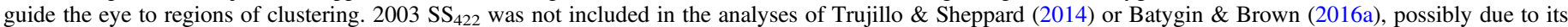
large $a$ uncertainty ( $\Delta a \sim 50 \mathrm{au}$ ); $2003 \mathrm{SS}_{422}$ is plotted in light blue in all panels and indicated in panel (a).

an observation bias. Trujillo \& Sheppard (2014) suggest that the clustering of TNOs with $\omega=0^{\circ}$ might be explainable through a dynamical interaction with a massive perturber on a 250 au orbit (required to maintain the observed clustering over time); they posit the existence of a ninth planet of super-Earthmass size in the outer solar system as the cause of the observed $\omega$ clustering, but they do not demonstrate a particular dynamical pathway to cause clustering around $\omega=0$ lasting until the present day.

In 2016, Batygin \& Brown (2016a) reexamined the $a>150$ au TNOs, reporting that the large- $a$ TNOs that are not dynamically interacting with Neptune show clustering not only in $\omega$ but also in longitude of the ascending node $\Omega$ and thus also in longitude of pericenter, $\varpi=\omega+\Omega$ (see Figure 1). A ninth planet (henceforth P9) with $a=700 \mathrm{au}$, eccentricity $e=0.6$, inclination $i=30^{\circ}$, and a mass of at least 10 Earth masses ${ }^{5}$ is proposed as the cause of the observed confinement in $\varpi$. They demonstrate analytically and with numerical simulations that their proposed P9 can produce $\varpi$ confinement for the age of the solar system in test particles with $a>250$ au and $\omega$ and $\Omega$ confinement for test particles with $a>500 \mathrm{au}$. They also find that an inclined P9 produces highly inclined distant TNOs, and they propose that such a planet could explain the origin of the known large- $a$ TNOs with $i$ between $60^{\circ}$ and $150^{\circ}$ (e.g., Drac $2008 \mathrm{KV}_{42}$; Gladman et al. 2009) whose formation was not securely identified (Elliot et al. 2005; Gladman et al. 2009; Chen et al. 2016) but has been explained by a distant planet (Gomes et al. 2015). These arguments launched a flurry of discussions and studies on the origins, location, and implications of a ninth planet. The studies have covered formation and capture scenarios (Bromley \& Kenyon 2016; Cowan et al. 2016; Kenyon \& Bromley 2016; Li \& Adams 2016; Mustill et al. 2016), constraints on the location, detectability and physical properties of P9 (Brown \& Batygin 2016; de la Fuente Marcos \& de la Fuente Marcos 2016a, 2016b; Fienga et al. 2016; Fortney et al. 2016; Ginzburg et al. 2016; Holman \& Payne 2016a,

\footnotetext{
5 Any distant planet that is interior to the Oort Cloud must have a mass less than $M_{\text {Saturn }}$ to be consistent with a nondetection in the all-sky WISE survey (Luhman 2014)
}

2016b; Linder \& Mordasini 2016; Philippov \& Chobanu 2016; Toth 2016; Veras 2016), the dynamical implications of P9 in the solar system (de la Fuente Marcos et al. 2016c; Lawler et al. 2016), P9 producing inclined TNOs (Batygin \& Brown 2016b), resonances and P9 (Beust 2016; Malhotra et al. 2016), a dark matter P9 (Sivaram et al. 2016), and impacts of P9 on the Sun's obliquity (Bailey et al. 2016; Lai 2016).

The studies to date have primarily focused on the formation and detection of $\mathrm{P} 9$, with few publications examining the impact on the observed TNO populations of a massive perturber in the distant solar system. Lawler et al. (2016) model the emplacement of the scattering and detached TNO populations in the presence of a super-Earth planet beyond $200 \mathrm{au}$. They find that such a planet sculpts distinctly different $e$ and $i$ distributions within the scattered and detached TNOs, but that this effect is not detectable in well-characterized, published surveys to date, due to the strong flux bias at those distances. De la Fuente Marcos et al. (2016c) performed numerical integrations of the six known $a>250$ au TNOs (see Table 3) in the presence of the four giant planets and the Batygin \& Brown (2016a) P9. Integrating these TNOs for 200 Myr, de la Fuente Marcos et al. (2016c) find that P9 may destabilize the orbits of several of these TNOs on timescales of dozens of megayears and can result in their ejection from the solar system. These two works are the first to study the implications of P9 on observed TNO populations, but to date there has been no work examining the implications on the detectability of the large- $a$ TNOs that were used to infer the existence of P9.

In this work we study the dynamical impact of P9 on the known large- $a$, large- $q$ TNOs (Table 1). Performing a set of $n$ body simulations, we examine the implications for the detectability of these TNOs and the evolution of their $\omega, \Omega$, and $\varpi$ angles to assess whether the proposed P9 reproduces the original observed clustering signal used to infer its existence.

\section{METHODS}

We test the P9 hypothesis by supposing the existence of such a planet in the solar system today and exploring the implications for the large- $a$ TNOs. We perform a set of $n$ - 
Table 1

All TNOs with $a>150$ au and $q>30$ au in the MPC Database

\begin{tabular}{|c|c|c|c|c|c|c|c|c|}
\hline $\begin{array}{l}\text { MPC } \\
\text { Designation }\end{array}$ & $\begin{array}{c}a \\
(\mathrm{au})\end{array}$ & $e$ & $\begin{array}{c}q \\
(\mathrm{au})\end{array}$ & $\begin{array}{c}i \\
(\mathrm{deg})\end{array}$ & $\begin{array}{c}\omega \\
(\mathrm{deg})\end{array}$ & $\begin{array}{c}\Omega \\
(\operatorname{deg})\end{array}$ & $\begin{array}{c}M \\
(\mathrm{deg})\end{array}$ & $H_{v}$ \\
\hline Sedna & 499.08 & 0.85 & 76.04 & 11.9 & 311.5 & 144.5 & 358.1 & 1.6 \\
\hline $2007 \mathrm{TG}_{422}$ & 482.4 & 0.93 & 35.57 & 18.6 & 285.8 & 112.9 & 0.4 & 6.2 \\
\hline $2010 \mathrm{~GB}_{174}$ & 369.73 & 0.87 & 48.76 & 21.5 & 347.8 & 130.6 & 3.3 & 6.5 \\
\hline $2013 \mathrm{RF}_{98}$ & 325.1 & 0.89 & 36.29 & 29.6 & 316.5 & 67.6 & 0.1 & 8.6 \\
\hline $2004 \mathrm{VN}_{112}$ & 317.71 & 0.85 & 47.32 & 25.6 & 327.1 & 66.0 & 0.4 & 6.4 \\
\hline $2012 \mathrm{VP}_{113}$ & 260.81 & 0.69 & 80.27 & 24.1 & 292.8 & 90.8 & 3.3 & 4.0 \\
\hline $2001 \mathrm{FP}_{185}$ & 226.86 & 0.85 & 34.26 & 30.8 & 7.0 & 179.3 & 1.3 & 6.2 \\
\hline $2000 \mathrm{CR}_{105}$ & 226.14 & 0.8 & 44.29 & 22.7 & 317.2 & 128.3 & 5.4 & 6.3 \\
\hline $2002 \mathrm{~GB}_{32}$ & 217.9 & 0.84 & 35.34 & 14.2 & 37.0 & 177.0 & 0.3 & 7.8 \\
\hline $2003 \mathrm{SS}_{422}$ & 196.44 & 0.8 & 39.37 & 16.8 & 210.8 & 151.1 & 359.2 & 7.1 \\
\hline $2007 \mathrm{VJ}_{305}$ & 187.74 & 0.81 & 35.18 & 12.0 & 338.3 & 24.4 & 1.5 & 6.6 \\
\hline $2003 \mathrm{HB}_{57}$ & 165.36 & 0.77 & 38.1 & 15.5 & 10.9 & 197.8 & 1.3 & 7.4 \\
\hline $2015 \mathrm{SO}_{20}$ & 162.02 & 0.8 & 33.16 & 23.4 & 354.9 & 33.6 & 359.8 & 6.4 \\
\hline $2013 \mathrm{GP}_{136}$ & 153.33 & 0.73 & 41.11 & 33.5 & 42.2 & 210.7 & 356.2 & 6.6 \\
\hline $2010 \mathrm{VZ}_{98}$ & 151.89 & 0.77 & 34.32 & 4.5 & 313.9 & 117.4 & 357.8 & 5.1 \\
\hline $2005 \mathrm{RH}_{52}$ & 151.21 & 0.74 & 38.98 & 20.5 & 32.3 & 306.1 & 2.6 & 7.8 \\
\hline
\end{tabular}

Note. Data downloaded on 2016 May 3. J2000 heliocentric orbital elements and absolute magnitudes are as reported by the MPC.

body integrations of the large- $a$ TNOs (see Figure 1, Table 1) in the presence of the giant planets and a candidate P9, examining the implications of the presence of a massive perturber through the orbital evolution of the TNOs. Batygin $\&$ Brown (2016a) report possible $a, e, i, \omega$, and $\Omega$ values for P9 (see Table 4) and assume a mass of around 10 Earth masses. The mean anomaly $\mathcal{M}$ of $\mathrm{P} 9$ is not well constrained by observations. A more distant P9 (i.e., $\mathcal{M}$ near $180^{\circ}$ ) is more difficult to detect, and thus more consistent with the current nondetection. Resonance confinement is not the proposed mechanism for $\varpi$ shepherding between P9 and the large- $a$ TNOs, and thus the choice of $\mathcal{M}$ does not affect the confinement; we therefore assign P9 $\mathcal{M}=180^{\circ}$. Batygin \& Brown (2016a) propose an $i=30^{\circ}$ for $\mathrm{P} 9$, motivated by this configuration's ability to produce highly inclined TNOs. We test the sensitivity of our analysis to this choice of inclination by also examining scenarios with $\mathrm{P} 9$ at lower inclinations of $15^{\circ}$ and $0^{\circ}$. Table 4 lists the orbital elements and mass for the three P9 configurations examined in this work; these parameters are consistent with current studies on the observable constraints (Brown \& Batygin 2016; Fienga et al. 2016; Holman \& Payne 2016a, 2016b). We also perform a control simulation without a P9.

Trujillo \& Sheppard (2014) note an $\omega$-clustering for TNOs with $a>150 \mathrm{au}$ and $q>30 \mathrm{au}$, and Batygin \& Brown (2016a) note that a subset of these $(a>250$ au and $q>30$ au) cluster in $\Omega$ and $\varpi$. The choice of a semimajor-axis boundary at 150 au has not been given any physical motivation; however, there is an apparent clustering of $\omega$ beyond this $a>150$ au threshold (Figure 1(a)), and so we continue this approach and select all of the TNOs satisfying $a>150$ au and $q>30$ au as the sample for this study ${ }^{6}$ for consistency and reproducibility with prior studies (Trujillo \& Sheppard 2014; Batygin \& Brown 2016a). Table 1 lists this set of TNOs with their orbital elements and absolute magnitudes $H$ as reported by the MPC.

We perform $n$-body simulations with the MERCURY6 (Chambers 1999) suite. The hybrid symplectic/Bulirsch-Stoer

\footnotetext{
6 The sample was selected in 2016 May.
}

algorithm, which balances integration speed with modeling close encounters, was used with a base time step of $0.5 \mathrm{yr},{ }^{7}$ and all simulations were run for $4 \mathrm{Gyr}^{8}$ An ejection distance of $10,000 \mathrm{au}$ is used. While TNOs that go beyond $a=10,000 \mathrm{au}$ can survive in the Oort Cloud, once they go beyond this distance in our simulation, they are no longer pertinent to the discussions in this work and so are removed for computational expediency. Galactic tides ${ }^{9}$ and stellar flybys are not included in the simulation.

The giant planets and TNOs in Table 1 were added to the simulation with orbital elements taken from the NASA Horizons database ${ }^{10}$ for the date of 2016 January 1 (JD $2,457,388.5)$. As $N$-body dynamics are inherently chaotic, we examine the evolution of each TNO through the evolution of a set of 60 clones created by sampling each object's orbit uncertainties. Orbit uncertainties for each TNO were taken from the JPL Small-body Database ${ }^{11}$ (Table 2), and clones were generated in one of two ways: (1) if the $a$ uncertainty was small ( $\Delta a<0.5 \mathrm{au}), 60$ orbits within the $a, e, i, \omega, \Omega$, and true anomaly uncertainties were randomly and uniformly sampled; (2) if $\Delta a>0.5 \mathrm{au}$, three clusters of orbits were generated-one cluster of 20 orbits at each of the $a, q$ extremes and one cluster of 20 orbits around the nominal orbit. For clarity we emphasize that in our simulation all TNO and planet orbits and uncertainties are taken from the NASA Horizons database and not the MPC; Table 1 lists the Minor Planet Center (MPC) elements and magnitudes as a convenient reference. We test three cases of P9 inclinations (Table 4) and one control simulation with the four giant planets. Each of our simulations thus includes four or five planets and 960 test

\footnotetext{
$70.5 \mathrm{yr}$ is less than $1 / 20$ of Jupiter's orbital period, which sets the shortest dynamical timescale in the simulation.

8 An accuracy parameter of 1 E-12 was used. Simulations consistently had a fractional energy change due to the integrator of order $3 \mathrm{E}-07$ and a fractional angular momentum change of $9 \mathrm{E}-10$.

9 While tides can affect the eccentricity of TNOs on Sedna-like orbits by a few percent (Veras \& Evans 2013), the effects of a giant planet in this TNO region can change eccentricities by tens of percent.

${ }^{10} \mathrm{http}: / /$ ssd.jpl.nasa.gov/horizons.cgi

11 http://ssd.jpl.nasa.gov/sbdb.cgi
} 
Table 2

Uncertainties in Orbital Parameters for All TNOs in Table 1

\begin{tabular}{lrcccc}
\hline \hline $\begin{array}{l}\text { MPC } \\
\text { Designation }\end{array}$ & $\begin{array}{c}\Delta a \\
(\mathrm{au})\end{array}$ & $\Delta e$ & $\begin{array}{c}\Delta i \\
(\mathrm{deg})\end{array}$ & $\begin{array}{c}\Delta \omega \\
(\mathrm{deg})\end{array}$ & $\begin{array}{c}\Delta \Omega \\
(\mathrm{deg})\end{array}$ \\
\hline Sedna & 0.78 & $2.39 \mathrm{e}-4$ & $3.04 \mathrm{e}-05$ & $1.34 \mathrm{e}-2$ & $1.60 \mathrm{e}-3$ \\
$2007 \mathrm{TG}_{422}$ & 3.45 & $5.24 \mathrm{e}-4$ & $2.45 \mathrm{e}-4$ & $2.93 \mathrm{e}-2$ & $1.74 \mathrm{e}-3$ \\
$2010 \mathrm{~GB}_{174}$ & 28.30 & $1.10 \mathrm{e}-2$ & $5.42 \mathrm{e}-3$ & $3.79 \mathrm{e}-1$ & $2.09 \mathrm{e}-2$ \\
$2013 \mathrm{RF}_{98}$ & 36.67 & $1.46 \mathrm{e}-2$ & $7.77 \mathrm{e}-2$ & 5.54 & $1.26 \mathrm{e}-1$ \\
$2004 \mathrm{VN}_{112}$ & 1.07 & $4.85 \mathrm{e}-4$ & $3.69 \mathrm{e}-4$ & $1.04 \mathrm{e}-2$ & $6.79 \mathrm{e}-4$ \\
$2012 \mathrm{VP}_{113}$ & 7.13 & $1.08 \mathrm{e}-2$ & $4.15 \mathrm{e}-3$ & 2.40 & $9.96 \mathrm{e}-3$ \\
$2001 \mathrm{FP}_{185}$ & 0.32 & $2.10 \mathrm{e}-4$ & $4.74 \mathrm{e}-4$ & $1.19 \mathrm{e}-2$ & $1.05 \mathrm{e}-4$ \\
$2000 \mathrm{CR}_{105}$ & 0.53 & $4.54 \mathrm{e}-4$ & $4.01 \mathrm{e}-4$ & $9.88 \mathrm{e}-3$ & $2.16 \mathrm{e}-4$ \\
$2002 \mathrm{~GB}_{32}$ & 0.68 & $4.92 \mathrm{e}-4$ & $2.27 \mathrm{e}-4$ & $3.82 \mathrm{e}-3$ & $3.53 \mathrm{e}-4$ \\
$2003 \mathrm{SS}_{422}$ & 47.83 & $5.56 \mathrm{e}-2$ & $5.89 \mathrm{e}-2$ & 17.18 & $6.98 \mathrm{e}-2$ \\
$2007 \mathrm{VJ}_{305}$ & 0.55 & $5.25 \mathrm{e}-4$ & $8.47 \mathrm{e}-4$ & $4.58 \mathrm{e}-2$ & $1.31 \mathrm{e}-3$ \\
$2003 \mathrm{HB}_{57}$ & 0.58 & $7.73 \mathrm{e}-4$ & $9.66 \mathrm{e}-4$ & $5.48 \mathrm{e}-2$ & $3.96 \mathrm{e}-4$ \\
$2015 \mathrm{SO}_{20}$ & 0.13 & $1.62 \mathrm{e}-4$ & $6.24 \mathrm{e}-4$ & $2.18 \mathrm{e}-2$ & $2.84 \mathrm{e}-4$ \\
$2013 \mathrm{GP}_{136}$ & 0.57 & $1.17 \mathrm{e}-3$ & $1.30 \mathrm{e}-3$ & $1.14 \mathrm{e}-1$ & $1.61 \mathrm{e}-4$ \\
$2010 \mathrm{VZ}_{98}$ & 0.15 & $2.10 \mathrm{e}-4$ & $6.11 \mathrm{e}-05$ & $7.58 \mathrm{e}-3$ & $3.57 \mathrm{e}-3$ \\
$2005 \mathrm{RH}_{52}$ & 0.19 & $2.91 \mathrm{e}-4$ & $5.90 \mathrm{e}-4$ & $6.84 \mathrm{e}-2$ & $1.48 \mathrm{e}-3$ \\
\hline & & & & &
\end{tabular}

Note. $1 \sigma$ uncertainties taken from the JPL small-body database (http://ssd.jpl. nasa.gov/sbdb.cgi). All values are J2000 heliocentric, generated for the JD 2,457,600.5 (2016 July 31).

particles; each was integrated for the $4 \mathrm{Gyr}$ age of the solar system. $^{12}$

\section{RESULTS}

\subsection{Signal in Angle Clustering}

P9 was proposed to explain the apparent clustering of one or more of the angles that determine an orbit's orientation. Here we examine the shepherding of $\omega, \Omega$, and $\varpi$ through our dynamical simulations of the large- $a$ TNOs. We focus our discussion of the results on the nominal P9 case with an inclination of $30^{\circ}$, checking that our results hold for different choices of P9 inclination (see below).

Here we examine the evolution of two of the TNOs in the sample as examples of the evolutions seen in our simulations. ${ }^{13}$ Figure 2 shows the orbital evolution for all of the clones of Sedna. The majority of Sedna clones undergo $\varpi$ shepherding (i.e., driven confinement in a band) throughout the $4 \mathrm{Gyr}$ simulation (Figure $2(\mathrm{~g})$ ), remaining in the region of the detected sample (red band). The orbital evolution of 2010 $\mathrm{GB}_{174}$ is plotted in Figure 3, showing that $2010 \mathrm{~GB}_{174}$ undergoes similar $\varpi$ shepherding. Of the 16 TNOs in the sample (Table 1), only six TNOs undergo $\varpi$ shepherding. These confinements last for periods ranging from hundreds of megayears to 4 Gyr (e.g., see Figures $2(\mathrm{~g})$ and $3(\mathrm{~g})$ ). We find that only TNOs with $a>250$ au (see Table 3) undergo $\varpi$ shepherding. The analysis of Batygin \& Brown (2016a) highlights both the set of $a>250$ au TNOs and six stable TNOs (see Table 3). We find that for TNOs with $q$ beyond Neptune, a large semimajor axis is the determining factor in $\varpi$ shepherding. All of the clones of the $a>250$ au TNOs experience shepherding; however, we find that two of the Batygin \& Brown (2016a) identified stable TNOs do not

\footnotetext{
12 Throughout, by "age of the solar system" we mean that we conduct a 4 Gyr integration, which demonstrates the behavior of the system for a length of time approximately equivalent to the age of the solar system.

${ }^{13}$ For plots of all TNOs across all simulations, see https://doi.org/10.5281/ zenodo.168604.
}

undergo this shepherding. A massive, eccentric, external perturber generically drives $q$ down to Neptune coupling (Figures 2(c) and 3(c)), which then shuts off $\varpi$ shepherding.

As a necessary implicit condition of $\varpi$ shepherding, P9 also drives the evolution of $\omega$ and $\Omega$ (Figures 2 and 3, panels (e) and (f)). Clones for all six of the $a>250$ au TNOs (Table 3) undergo $\varpi$ shepherding and thus experience correlated $\omega$ and $\Omega$ evolution, but only two $\left(\mathrm{VP}_{113}\right.$ and $\left.\mathrm{GB}_{174}\right)$ experience shepherding of $\omega$ and $\Omega$, while the rest drift in a pattern that is mostly consistent with long-term circulation at a rate unique to each TNO. Whether their $\omega$ and $\Omega$ are shepherded or circulating, the clones experience $\varpi$ confinement in the same region.

Figure 4 explores the implication of this evolution on the initial signal used to infer the existence of P9: simultaneous $\omega$, $\Omega$, and $\varpi$ clustering. The clones in Figure 4 are color-coded red if they are in the region of $\varpi$ confinement at $2 \mathrm{Gyr}^{14}$ into the simulation (panel (c)) and blue otherwise. Panels (a) and (b) show that clones in the $\varpi$ confinement band can occupy all values in $\omega$ and $\Omega$. This is consistent with the result that the explored distant massive planets do not shepherd $\omega$ or $\Omega$ in the region of the observed TNOs (Batygin \& Brown 2016a; Lawler et al. 2016). Panel (c) shows that while some clones experience $\varpi$ shepherding (Figures 2 and 3), the influence of P9 does not sculpt a dominant band of confined $\varpi$ values. Figure 5 presents an alternative visualization of these results. The lack of sculpting implies that there should be a large number of detectable TNOs at all values of $\omega, \Omega$, and $\varpi$.

\subsection{Orbital Evolution}

A $10 M_{\oplus}$ planet with its $q$ at 280 au has a significant effect on the orbital evolution of the TNOs in our sample. In their explorations of the $a>250$ au TNOs, de la Fuente Marcos et al. (2016c) demonstrate that P9 can destabilize the orbit of these TNOs on short timescales $(<200 \mathrm{Myr})$; we extend the sample to include all TNOs with $a>150$ au and $q>30 \mathrm{au}$, and we also extend simulations to the age of the solar system, revealing important structure in the $i$ and $q$ evolution of these TNOs.

\subsubsection{Perihelia Cycling}

Gravitational interactions with P9 raise and lower the pericenters of all of the clones in the sample, lowering some clones down into Neptune, or even Jupiter, crossing orbits or raising them out to hundreds of au (e.g., see Figures 2 and 3). All of the $\varpi$-shepherded clones undergo $q$ oscillations, which occur on roughly the same timescale as their $\varpi$ oscillations (see Figures 2(c) and 3(c)). With $q$ cycled between hundreds of au and tens of au, the idea of a gap in overall distribution of TNO perihelia in the 50-70 au range (Trujillo \& Sheppard 2014) is incompatible with the P9 hypothesis. The cycling $q$ affects the stability of the clones as they are pushed into planet-crossing orbits, and thus affects their survivability on gigayear timescales. This increases the likelihood of ejection for the clones of TNOs that already currently interact with Neptune, and it introduces instability for those that are currently on Neptune decoupled orbits (e.g., Sedna, 2012 VP113). Table 5 gives the fraction of clones ejected for each TNO in our sample across the four simulations performed. In contrast, the cycling to high

\footnotetext{
${ }^{14} 2 \mathrm{Gyr}$ is taken as a representative snapshot for visualization, but the results does not depend on epoch choice.
} 

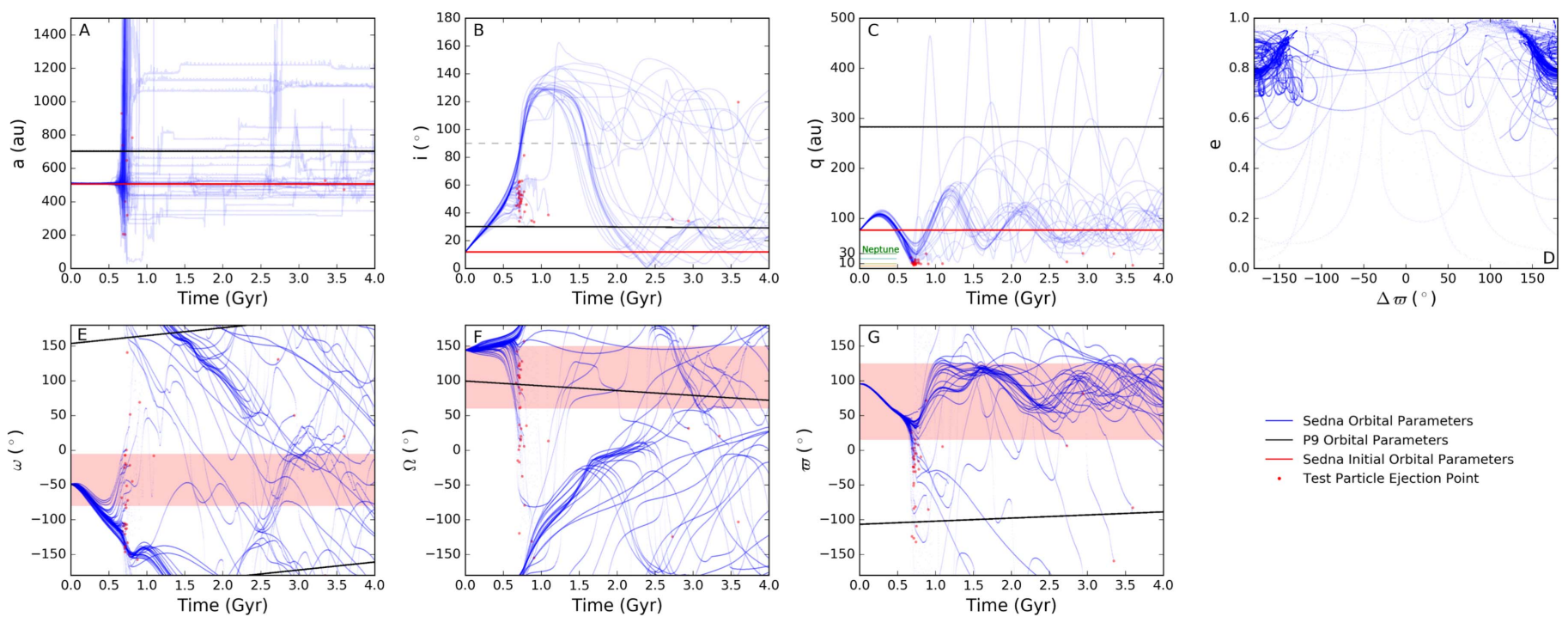

Figure 2. Orbital evolution for the barycentric orbital elements of the 60 clones of Sedna across the 4 Gyr simulation. The time evolutions of clones are plotted in blue in all panels. Panel (d) plots the $\varpi_{\mathrm{P} 9}-\varpi_{\text {clone }}$ vs. $e$, which shows the secular interaction between the clones and P9. The red horizontal lines in panels (a), (b), and (c) mark the present-day observed values for Sedna's orbital elements plotted in those panels. The black line plots the orbital evolution of P9. Red circles mark the ejection point of a clone from the simulation ( $a>10,000$ au or collision with the Sun). The red bands in panels (e), (f), and (g) mark the region of confinement in the real TNOs for $\omega, \Omega$, and $\varpi$, respectively (as in Figure 1). The dashed gray line in panel (b) indicates an inclination of $90^{\circ}$. The orbits of the giant planets are plotted in panel (c), showing when clones are driven into giant-planet-crossing orbits. The feature in panel (a) near 0.75 Gyr results from strong scattering encounters between the test particles and the giant planets, which can be seen in panel (c).
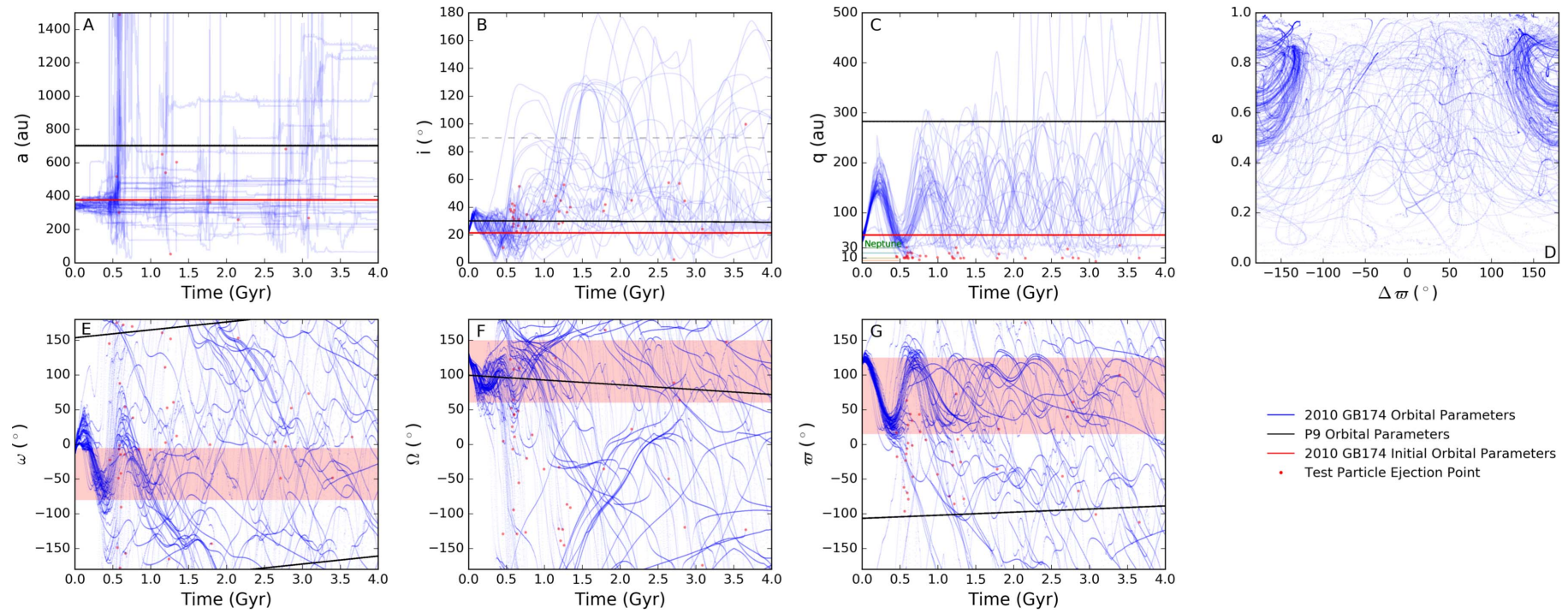

Figure 3. Orbital evolution for the 60 clones of $2010 \mathrm{~GB}_{174}$ across the $4 \mathrm{Gyr}$ simulation. Line styles and panels are as described in Figure 2.

Table 3

Examined TNO Groups

\begin{tabular}{|c|c|c|c|c|c|c|}
\hline \multirow{2}{*}{$\begin{array}{l}\text { Category } \\
a>250 \mathrm{au}\end{array}$} & \multicolumn{6}{|c|}{ Designation } \\
\hline & Sedna & $2010 \mathrm{~GB}_{174}$ & $2004 \mathrm{VN}_{112}$ & $2012 \mathrm{VP}_{113}$ & $2007 \mathrm{TG}_{422}$ & $2013 \mathrm{RF}_{98}$ \\
\hline Batygin \& Brown (2016a) identified stable & Sedna & $2010 \mathrm{~GB}_{174}$ & $2004 \mathrm{VN}_{112}$ & $2012 \mathrm{VP}_{113}$ & $2000 \mathrm{CR}_{105}$ & $2010 \mathrm{VZ}_{98}$ \\
\hline
\end{tabular}

values reduces the detectability of the clones. This then requires a larger than previously expected reservoir of large- $a$ TNOs to explain the detection of the currently observed sample.

\subsubsection{Sedna Population Estimate}

We examine the implications for the detection of Sedna (the most massive of the known $a>150$ au TNOs) in detail as an example of the effects P9 induces on $q$ and $i$ evolution on the large- $a$ TNOs. Of the 60 clones of Sedna, 63\% of the clones are ejected during the simulation (see Table 5). For the clones of Sedna that were not ejected, each spent on average $\sim 45 \%$ of the $4 \mathrm{Gyr}$ simulation with $q$ beyond the limit of detectability (of Sedna's discovery survey) due to $q$ raising, implying a large population of undetectable large- $a$ Sedna-like TNOs. As Sedna 

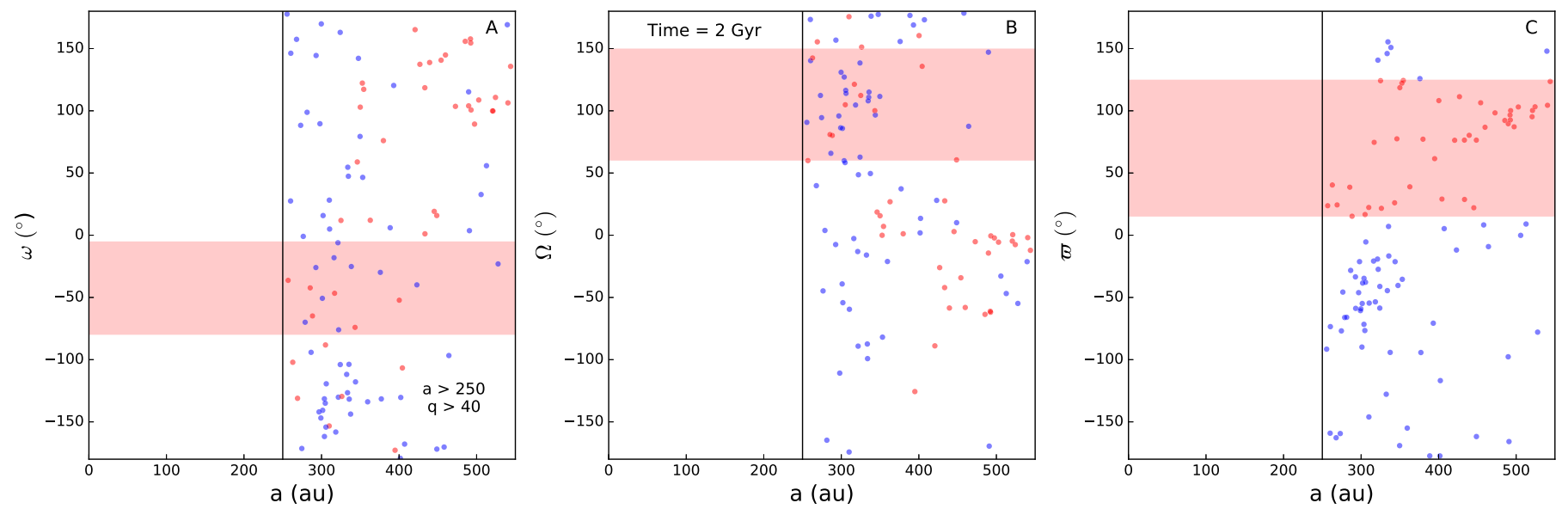

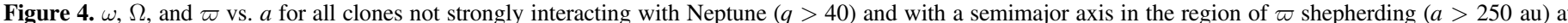

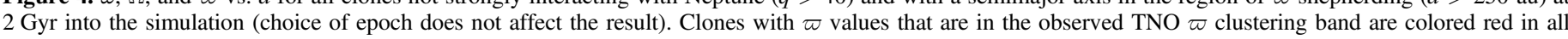

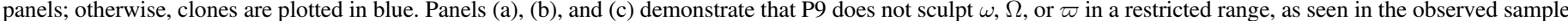

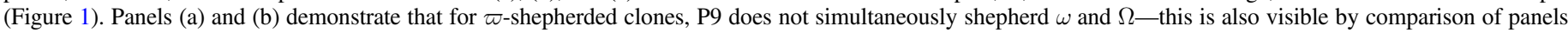
(e) and (f) in Figures 2 and 3.

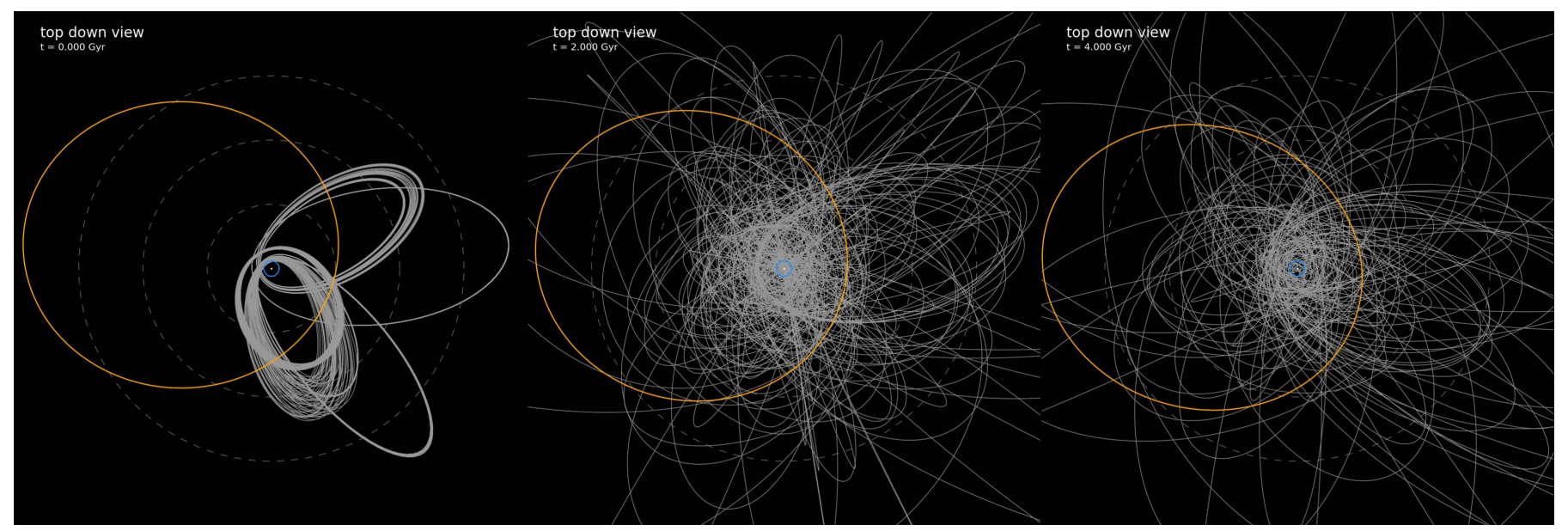

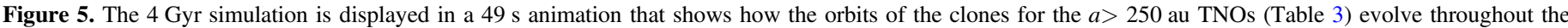

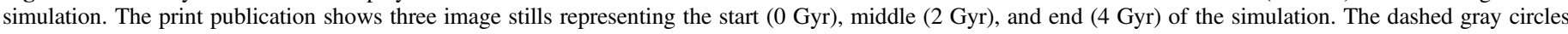

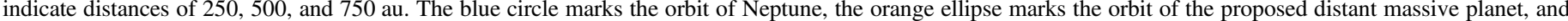

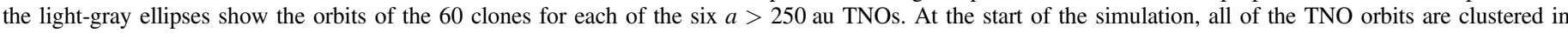

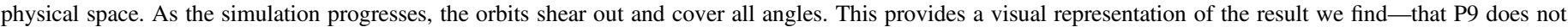
sculpt a confined alignment of orbits, even with the alignment present in the starting conditions.

(An animation of this figure is available.)

Table 4

P9 Configurations Tested

\begin{tabular}{|c|c|c|c|c|c|c|c|c|}
\hline P9 & $\begin{array}{c}a \\
(\mathrm{au})\end{array}$ & $e$ & $\begin{array}{c}q \\
(\mathrm{au})\end{array}$ & $\begin{array}{c}i \\
(\operatorname{deg})\end{array}$ & $\begin{array}{c}\omega \\
(\operatorname{deg})\end{array}$ & $\begin{array}{c}\Omega \\
(\operatorname{deg})\end{array}$ & $\begin{array}{c}M \\
(\mathrm{deg})\end{array}$ & $\begin{array}{l}\text { Mass } \\
\left(M_{\oplus}\right)\end{array}$ \\
\hline P9 (Batygin \& Brown 2016a) & 700 & 0.6 & 280 & 30 & 150 & 100 & 180 & 10 \\
\hline P9 (moderate $i$ ) & 700 & 0.6 & 280 & 15 & 150 & 100 & 180 & 10 \\
\hline P9 (low $i)$ & 700 & 0.6 & 280 & 0 & $\cdots$ & $\cdots$ & 180 & 10 \\
\hline
\end{tabular}

Note. With an inclination of $0^{\circ}, \omega$ and $\Omega$ become undefined, but $\varpi$ remains $150^{\circ}$.

clones spend on average $\sim 45 \%$ of the simulation with $q$ beyond the detection limit, roughly one-half of the population must be on completely undetectable orbits today.

The shape of an orbit, which sets the fraction of the orbit inside the detectable volume of surveys, affects the expected number of TNOs in the population that are required to explain the detected sample. The detection of a TNO like Sedna that is on such a large- $a$ orbit, where most of the orbit lies beyond a detection threshold, implies that there must be many TNOs on similar orbits in order for it to be probable to have detected Sedna near its pericenter. Estimates of the size of these populations are regularly computed by asking what fraction of 
Table 5

Fraction of Clones Ejected during the Simulation for the Different P9 Inclinations Tested

\begin{tabular}{lcccc}
\hline \hline & \multicolumn{4}{c}{ \% Ejected } \\
\cline { 2 - 5 } Designation & $30^{\circ}$ & $15^{\circ}$ & $0^{\circ}$ & No P9 \\
\hline Sedna & 63 & 37 & 7 & 0 \\
$2007 \mathrm{TG}_{422}$ & 67 & 95 & 82 & 88 \\
$2010 \mathrm{~GB}_{174}$ & 63 & 60 & 53 & 8 \\
$2013 \mathrm{RF}_{98}$ & 93 & 93 & 90 & 52 \\
$2004 \mathrm{VN}_{112}$ & 47 & 62 & 62 & 0 \\
$2012 \mathrm{VP}_{113}$ & 77 & 52 & 75 & 0 \\
$2001 \mathrm{FP}_{185}$ & 90 & 82 & 78 & 63 \\
$2000 \mathrm{CR}_{105}$ & 75 & 63 & 53 & 0 \\
$2002 \mathrm{~GB}_{32}$ & 88 & 85 & 83 & 82 \\
$2003 \mathrm{SS}_{422}$ & 82 & 83 & 77 & 42 \\
$2007 \mathrm{VJ}_{305}$ & 82 & 88 & 88 & 73 \\
$2003 \mathrm{HB}_{57}$ & 70 & 62 & 65 & 28 \\
$2015 \mathrm{SO}_{20}$ & 75 & 82 & 85 & 88 \\
$2013 \mathrm{GP}_{136}$ & 33 & 25 & 10 & 10 \\
$2010 \mathrm{VZ}_{98}$ & 68 & 93 & 90 & 93 \\
$2005 \mathrm{RH}_{52}$ & 63 & 40 & 38 & 17 \\
\hline
\end{tabular}

Note. The clones sample the range around plausible orbits for each TNO, but are not constructed to represent the possible orbits given the ephemerides. For this reason, this table should not be interpreted as giving the stability for the actual orbit of the TNOs in the sample.

the time each TNO spends in an observable part of its orbit, which then gives the number of TNOs required for the expected detection of one object (as was done for Sedna in Brown et al. 2004; Schwamb et al. 2009). We examine all of the clones of Sedna, at all time points in the simulation, and compute the population estimate required for the detection of those clones that have $q$ within the detection threshold. While detectable, the Sedna clones require a population of TNOs on their orbit ranging from tens to, at times, the high thousands. We find a mean population estimate of $\sim 80$ Sedna-sized objects (across all clones and all time steps) required for the detection of Sedna. This estimate is double the best estimate for the Sedna population (Schwamb et al. 2009) but within the $1 \sigma$ upper limit for their size of the Sedna population. We combine our mean population estimate (80) with our findings that less than half of the clones of Sedna survive, and that surviving clones are only visible during half of the $4 \mathrm{Gyr}$ simulation. In the P9 scenario, the single detection of Sedna today requires a mass of 6-24 $M_{\oplus}$ from the ensemble of Sedna-like TNOs (down to absolute magnitude ${ }^{15} H_{r}=8$ ). This is more than an order of magnitude greater than the mass estimate required for the case of no P9.

\subsubsection{Inclination Raising}

Perturbations from $\mathrm{P} 9$ raise and lower the inclinations of all TNOs in the sample. Figures 2(b) and 3(b) show that, on gigayear timescales, P9 raises the inclination, even into the retrograde state, before cycling them back to lower inclination.

\footnotetext{
15 The absolute magnitude, $H$, distribution transitions to a different form faint of $H_{r}=8$, which is diameter $D=60 \mathrm{~km}$ for $16 \%$ albedo. A single slope absolute magnitude distribution with slopes of 0.8 and 0.9 was used to determine the range of mass estimates. These slopes are consistent with the measurements of other TNO populations and the TAOS limit on the Sedna population (Wang et al. 2009). An albedo of $16 \%$ and a density of $1 \mathrm{~g} \mathrm{~cm}^{-3}$ were used for the mass estimates.
}

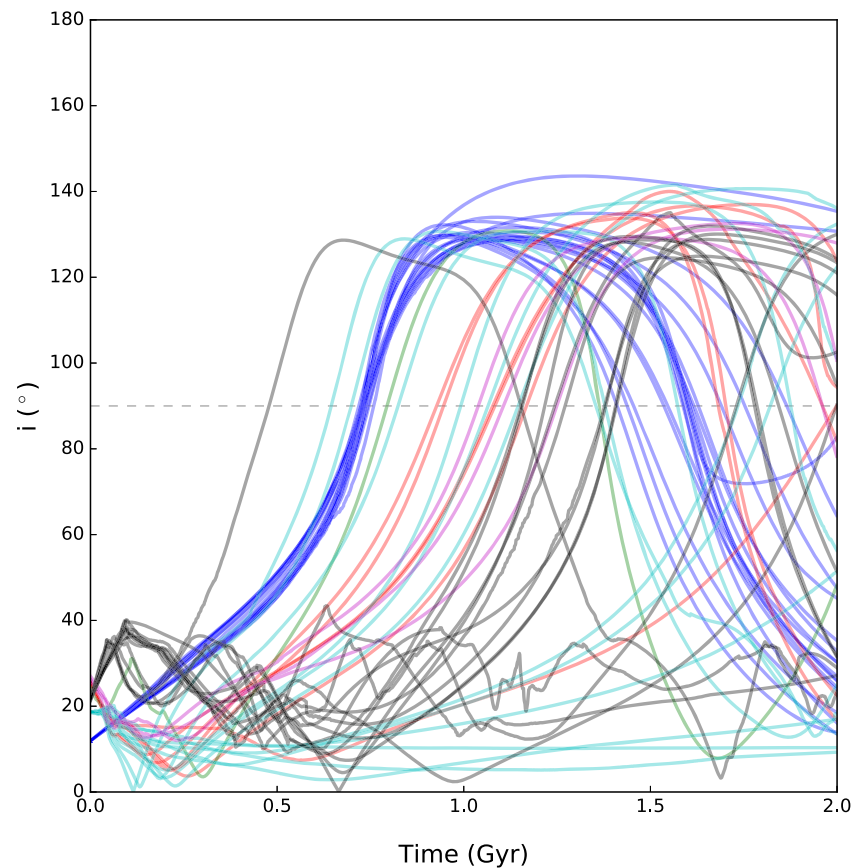

Figure 6. The $i$ evolution for all clones in our simulation that undergo $\varpi$ shepherding for the first 2 Gyr. Each color represents clones from one of the six $a>250$ au TNOs (Table 3). A dashed gray line marks $i=90^{\circ}$. The colorcoding serves to demonstrate that clones of each TNO undergo the same generic evolution of $i$ cycling, which goes through extreme and retrograde values. Raising inclinations through retrograde values is a generic feature of the dynamical mechanism that causes $\varpi$ shepherding.

Examining only the clones that undergo $\varpi$ shepherding, we find that this $i$ flipping is a characteristic feature from the secular interactions (see Figure 6). The P9 hypothesis implies the existence of an undetected, but potentially detectable, population of high- $i$ and retrograde large- $a$ TNOs. Clones spend over half of the time with $i>30^{\circ}$, suggesting that for each detected TNO there should be at least one undetected TNO at a higher inclination. This implies that in a P9 solar system, the mass estimates above should be factors of several larger.

We test the sensitivity of our results to the choice of P9 inclination. In all simulations, we find that $\varpi$ shepherding is only induced for $a>250$ au clones. Figure 7(b) shows that the $q$ oscillations occur across all choices of P9 $i$ for $\varpi$-shepherded clones. Inclination raising and flipping occurs for both P9 simulations with a nonzero $i$ perturber and takes the same form of raising inclinations through extreme and retrograde values that then cycle back to small values (Figure 7(a)). The results in this work are independent of the choice of P9 inclination, with the exception that a zero-degree inclination for P9 does not induce the same raising and flipping of inclinations in the clones of $\varpi$-shepherded TNOs.

\section{SUMMARY AND DISCUSSION}

1. The P9 scenarios explored do not generically induce $\omega$ shepherding for the region of the observed TNOs $(a<500 \mathrm{au})$, a fact already noted in Batygin \& Brown (2016a). This holds true even for TNOs that exhibit $\varpi$ shepherding. The apparent clustering of $\omega$ is the initial signal that motivated the current incarnation of the hypothesis of a distant planet in the solar system. The P9 

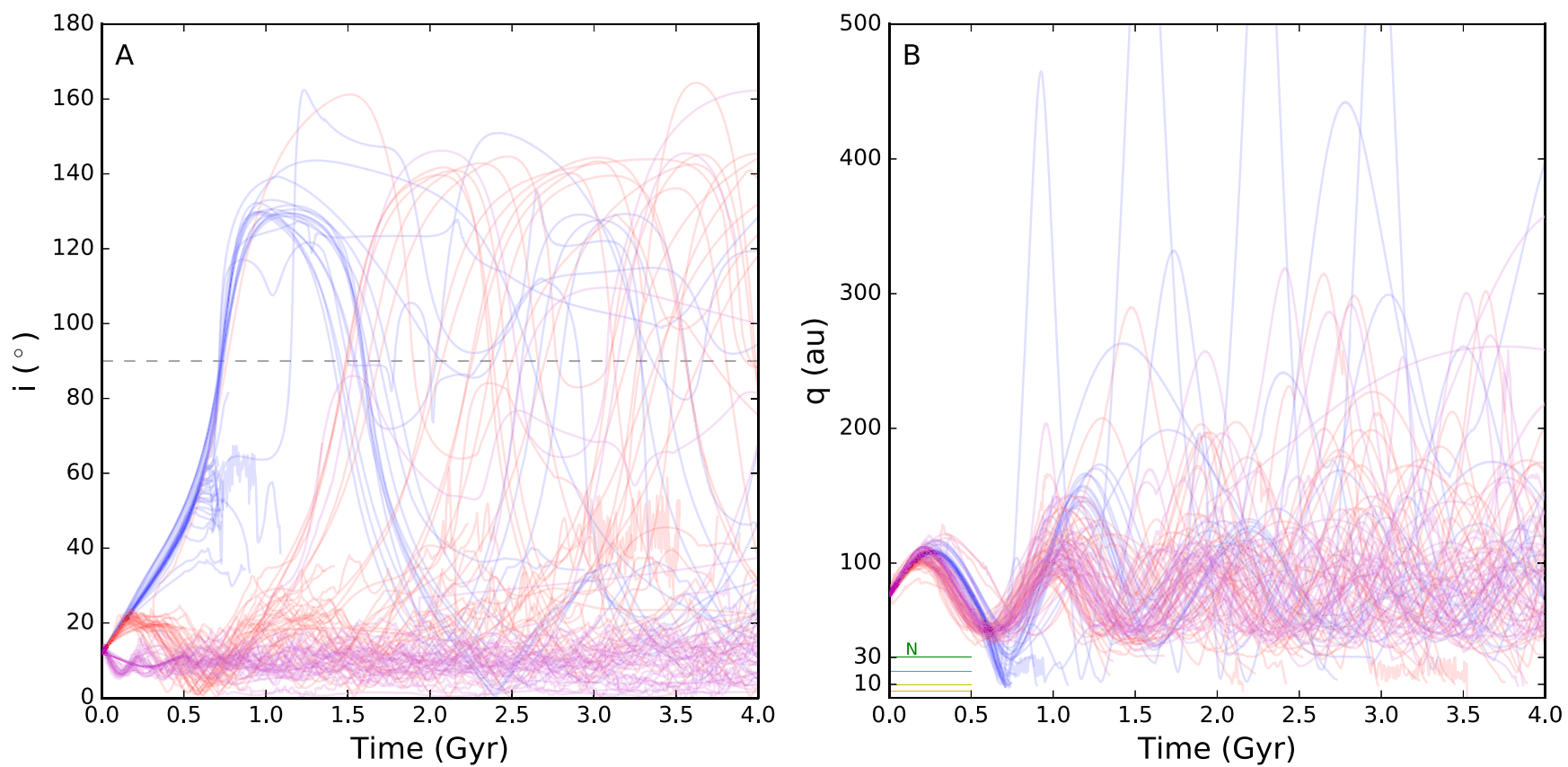

Figure 7. The $i, q$ evolution of the Sedna clones for the various $\mathrm{P} 9$ configurations. Clones from the simulation with $i=30^{\circ}$ are shown in blue, $\mathrm{P} 9 i=15^{\circ}$ in red, and P9 $i=0^{\circ}$ in magenta. A dashed gray line marks $i=90^{\circ}$ in panel (a). Panel (a) shows that the same $i$ raising and flipping is induced for $\varpi$-shepherded clones in both the $\mathrm{P} 9 i=30^{\circ}$ and $\mathrm{P} 9 i=15^{\circ}$ cases. Panel (b) shows that large- $q$ oscillations occur for $\varpi$-shepherded clones in all simulations. The cycling of $q$ is a generic feature of a massive external perturber, regardless of the perturber's inclination. Inclination raising occurs generically for $\varpi$ shepherded clones in the simulations with high $\left(30^{\circ}\right)$ and more moderate $\left(15^{\circ}\right)$ perturber inclinations.

scenarios explored in this work do not reproduce the observed signal of simultaneous clustering in $\omega, \Omega$, and $\varpi$ in the region of the detections (Figure 1).

2. Clones that undergo $\varpi$ shepherding have their inclinations lifted to retrograde values. If there is a massive distant ninth planet, then there should be a significant number of lower- $q$, large- $a$, large- $i$ (or even retrograde) TNOs; only two retrograde TNOs (2008 $\mathrm{KV}_{42}$ or Drac, Gladman et al. 2009; and $2011 \mathrm{KT}_{19}$ or Niku, Chen et al. 2016) are known today, both of which have small semimajor axes. Additionally, clones of TNOs that undergo $\varpi$ shepherding (i.e., those with $a>250$ au) spend a significant portion of the age of the solar system with large inclinations, but all of the large- $a$ TNOs were detected with $i<30^{\circ}$. While there is a bias toward detection at low $i$, Sedna was detected in an all-sky survey with sensitivity to high $i$, and other surveys have looked at high latitudes (e.g., Schwamb et al. 2009; NGVS, Chen et al. 2013; CFEPS, Petit et al. 2016), but none have found any large- $a(>100$ au), high- $i$ TNOs. The lack of detections of highly inclined TNOs poses a challenge for the inclined P9 scenario.

3. During their orbital evolutions, $\varpi$-shepherded clones evolve through $q>80$ au orbits, where they would not be detected. The existence of a few $q \sim 40-80$ au detections implies a very massive (tens of $M_{\oplus}$ ) distant- $q$ reservoir and thus an efficient mechanism for delivery of material into this distant zone of the solar system. Implanting $\gtrsim 10 M_{\oplus}$ of solids on large- $a$, large- $q$ orbits with a high efficiency in the range of $10 \%$ to $1 \%$ would require an implausibly large initial planetesimal disk with, respectively, hundreds to thousands of $M_{\oplus}$ of solids.

4. The existence of a distant massive planet destabilizes the orbits of the large- $a$ TNOs, like $2000 \mathrm{CR}_{105}, 2004 \mathrm{VN}_{112}$, and $2012 \mathrm{VP}_{113}$, that would otherwise remain stable for the age of the solar system. Such a scenario thus requires an active supply reservoir and mechanism to deliver objects onto these orbits. The perturber itself may act as the supply for this region, but this avenue was not explorable with these simulations. The pericenter raising of a distant massive perturber will necessarily populate orbits with $q$ between 50 and $70 \mathrm{au}$, and therefore the scenario is inconsistent with the suggestion of a gap in the $q$ distribution proposed in Trujillo \& Sheppard (2014).

5. As has been recently noted by Sheppard \& Trujillo (2016), some of the observed clustering in $\Omega$ may, in fact, be the result of observing bias. Given that the observed TNOs cluster in $\omega$ near 0 , and given a strong bias to detecting the TNO at pericenter, the $\Omega$ detected is determined by the direction of the survey pointing. The location of survey pointings is determined by galactic plane avoidance and local weather conditions that are season dependent. These biases may be able to explain the apparent clustering in $\Omega$ of the detected sample; future work is required to examine the effects of this bias in detail. While this manuscript was in preparation, Sheppard \& Trujillo (2016) reported the discovery of several new large- $a$ TNOs that would fall within the sample explored here. Two of these TNOs have $\Omega$ values that broaden the range in the observed sample, which suggests that this apparent clustering will be eroded with future detections.

A bias in $\Omega$, given the clustering in $\omega$, would cause apparent $\varpi$ clustering. In order to explain the observed sample, the successful theory must be able to explain either the observational bias or the dynamical pathway to shepherding $\omega$ that results in the apparent clustering of $\omega$ in the observed sample.

6. A massive external perturber generically causes cyclic pericenter oscillations that drive TNOs into Neptune- or 
even Jupiter-crossing orbits. This process decouples TNOs from any shepherding influence of the external perturber and results in the randomization of $\omega$ and $\Omega$ in the large- $a$ TNOs due to precession. TNOs interacting with Neptune and the external perturber can become distributed in the large- $a$ region with random $\omega$ and $\Omega$ angles and should be part of the detected sample. The observed clustering of $\omega$ and $\Omega$ (Figure 1) is not produced in the P9 scenario.

7. In exoplanet and debris disk systems with a massive external perturber, $\varpi$ shepherding may have implications for dust production. The induced shepherding of $\varpi$ aligns orbits in physical space and will bring particles to pericenter in the same angular region, which may enhance the collisional probability and thus dust production for this location. This may prove an interesting avenue to explore for systems with massive eccentric exoplanets beyond the debris disk and could possibly contribute to a pericenter glow (Wyatt et al. 1999).

\section{CONCLUSION}

In this work we have integrated clones of the $a>150 \mathrm{au}$, $q>30$ au TNOs for $4 \mathrm{Gyr}$ in the presence of a candidate P9 perturber, examining the consequences of a distant massive perturber on the TNOs used to infer the planet's existence. We find that P9 shepherds $\varpi$ for clones with $a>250$ au, driving this confinement for hundreds of megayears to $4 \mathrm{Gyr}$. Clones that experience $\varpi$ shepherding also undergo $q$ oscillations and $i$ flipping, which suggests the presence of a very massive (tens of $M_{\oplus}$ ) reservoir of large- $a$ TNOs. The P9 scenario produces a larger reservoir of potentially detectable yet unseen high- $i$ TNOs with shepherded $\varpi$ values, suggesting that there is a currently missing or unseen signature of P9. The P9 scenario does not produce the observed simultaneous clustering in the angles $\omega, \Omega$, and $\varpi$ that is seen in the detected sample. Taken alone, each of the consequences poses a challenge for the P9 scenario. Taken together, these consequences suggest that the existence of the proposed distant massive perturber is unlikely.

A distant massive perturber produces a set of very interesting signatures on a set of large- $a$ planetesimals, but the signature that has driven the newest incarnation of the distant planet hypothesis, namely, the clustering of $\omega, \Omega$, and $\varpi$, is not produced by this scenario. Ongoing surveys (like the Outer Solar Systems Origins Survey [Bannister et al. 2016] and that of Trujillo \& Sheppard 2014; Sheppard \& Trujillo 2016) will hopefully provide the detections and proper survey characterizations needed to examine the underlying impetus for the P9 scenario: the apparent clustering in $\omega, \Omega$, and $\varpi$ of the large$a$ TNOs.

This project was funded by the National Science and Engineering Research Council and the National Research Council of Canada. This research used the facilities of the Canadian Astronomy Data Centre operated by the National Research Council of Canada with the support of the Canadian Space Agency. C.S. gratefully acknowledges support from the
NSERC CGS Fellowship. S.M.L. gratefully acknowledges support from the NRC Canada Plaskett Fellowship.

Software: Mercury, Python.

\section{REFERENCES}

Bailey, E., Batygin, K., \& Brown, M. E. 2016, AJ, 152, 126

Bannister, M. T., Kavelaars, J. J., Petit, J.-M., et al. 2016, AJ, 152, 70

Batygin, K., \& Brown, M. E. 2016a, AJ, 151, 22

Batygin, K., \& Brown, M. E. 2016b, arXiv:1610.04992

Becker, A. C., Agol, E., Silvestri, N. M., et al. 2008, MNRAS, 386, 416

Beust, H. 2016, A\&A, 590, L2

Brasser, R., \& Schwamb, M. E. 2015, MNRAS, 446, 3788

Bromley, B. C., \& Kenyon, S. J. 2016, ApJ, 826, 64

Brown, M. E., \& Batygin, K. 2016, ApJL, 824, L23

Brown, M. E., Trujillo, C., \& Rabinowitz, D. 2004, ApJ, 617, 645

Chambers, J. E. 1999, MNRAS, 304, 793

Chen, Y.-T., Kavelaars, J. J., Gwyn, S., et al. 2013, ApJL, 775, L8

Chen, Y.-T., Lin, H. W., Holman, M. J., et al. 2016, ApJL, 827, L24

Cowan, N. B., Holder, G., \& Kaib, N. A. 2016, ApJL, 822, L2

de la Fuente Marcos, C., \& de la Fuente Marcos, R. 2016a, MNRAS, 459, L66

de la Fuente Marcos, C., \& de la Fuente Marcos, R. 2016b, MNRAS, 462, 1972

de la Fuente Marcos, C., de la Fuente Marcos, R., \& Aarseth, S. J. 2016c, MNRAS, 460, L123

Einstein, A. 1916, AnP, 354, 769

Elliot, J. L., Kern, S. D., Clancy, K. B., et al. 2005, AJ, 129, 1117

Fienga, A., Laskar, J., Manche, H., \& Gastineau, M. 2016, A\&A, 587, L8

Fortney, J. J., Marley, M. S., Laughlin, G., et al. 2016, ApJL, 824, L25

Ginzburg, S., Sari, R., \& Loeb, A. 2016, ApJL, 822, L11

Gladman, B., \& Chan, C. 2006, ApJL, 643, L135

Gladman, B., Holman, M., Grav, T., et al. 2002, Icar, 157, 269

Gladman, B., Kavelaars, J., Petit, J.-M., et al. 2009, ApJL, 697, L91

Gladman, B., Marsden, B. G., \& Vanlaerhoven, C. 2008, Nomenclature in the Outer Solar System (Tucson, AZ: Univ. Arizona Press)

Gomes, R. S. 2003, Icar, 161, 404

Gomes, R. S., Gallardo, T., Fernández, J. A., \& Brunini, A. 2005, CeMDA, 91, 109

Gomes, R. S., Matese, J. J., \& Lissauer, J. J. 2006, Icar, 184, 589

Gomes, R. S., Soares, J. S., \& Brasser, R. 2015, Icar, 258, 37

Holman, M. J., \& Payne, M. J. 2016a, AJ, 152, 80

Holman, M. J., \& Payne, M. J. 2016b, AJ, 152, 94

Ida, S., Larwood, J., \& Burkert, A. 2000, ApJ, 528, 351

Kaib, N. A., Roškar, R., \& Quinn, T. 2011, Icar, 215, 491

Kenyon, S. J., \& Bromley, B. C. 2004, Natur, 432, 598

Kenyon, S. J., \& Bromley, B. C. 2016, ApJ, 825, 33

Lai, D. 2016, arXiv:1608.01421

Lawler, S. M., Shankman, C., Kaib, N., et al. 2016, arXiv:1605.06575

Le Verrier, U. J. 1846, AN, 25, 65

Le Verrier, U. J. 1859, AnPar, 5, P1

Li, G., \& Adams, F. C. 2016, ApJL, 823, L3

Linder, E. F., \& Mordasini, C. 2016, A\&A, 589, A134

Luhman, K. L. 2014, ApJ, 781, 4

Lykawka, P. S., \& Mukai, T. 2007, Icar, 192, 238

Malhotra, R., Volk, K., \& Wang, X. 2016, ApJL, 824, L22

Morbidelli, A., \& Levison, H. F. 2004, AJ, 128, 2564

Mustill, A. J., Raymond, S. N., \& Davies, M. B. 2016, MNRAS, 460, L109

Petit, J., Kavelaars, J. J., Gladman, B. J., et al. 2016, arXiv:1608.02873

Philippov, J. P., \& Chobanu, M. I. 2016, PASA, 33, e033

Schwamb, M. E., Brown, M. E., \& Rabinowitz, D. L. 2009, ApJL, 694, L45

Sheppard, S. S., \& Trujillo, C. 2016, arXiv:1608.08772

Sivaram, C., Kenath, A., \& Kiren, O. V. 2016, Ap\&SS, 361, 230

Soares, J. S., \& Gomes, R. S. 2013, A\&A, 553, A110

Thommes, E. W., Duncan, M. J., \& Levison, H. F. 2002, AJ, 123, 2862

Toth, I. 2016, A\&A, 592, A86

Trujillo, C. A., \& Sheppard, S. S. 2014, Natur, 507, 471

Veras, D. 2016, MNRAS, 463, 2958

Veras, D., \& Evans, N. W. 2013, CeMDA, 115, 123

Wang, J.-H., Lehner, M. J., Zhang, Z.-W., et al. 2009, AJ, 138, 1893

Wyatt, M. C., Dermott, S. F., Telesco, C. M., et al. 1999, ApJ, 527, 918 